\title{
HUELLA ECOLÓGICA Y BIOCAPACIDAD: INDICADORES BIOFÍSICOS PARA LA GESTIÓN AMBIENTAL. El caso de Manizales, Colombia $\underline{(1)}$
}

\author{
Isaías Tobasura Acuña ${ }^{i}$ \\ ${ }^{i}$ Doctor en Sociología del Medio Ambiente. Profesor de la Universidad de Caldas, \\ Manizales, Colombia. E-mail: isaiast@epm.net.co
}

Manizales, 2008-05-05 (Rev. 2008-06-12)

\section{RESUMEN}

El bienestar de la población asentada en un territorio (un país, una ciudad, un pueblo) está íntimamente ligado a la capacidad de oferta de bienes y servicios ambientales. Conocer los requerimientos de la población y la capacidad del espacio disponible para la satisfacción de sus necesidades, es fundamental para garantizar la sustentabilidad ambiental. La Economía Ecológica nos permite, a través del cálculo de la Huella Ecológica HE y la Biocapacidad BC estimar el déficit ambiental de un territorio determinado. Aunque a nivel global la utilidad práctica de estos indicadores para la toma de decisiones es limitada, en los ámbitos nacional, regional o local son instrumentos útiles para la toma de decisiones en la planificación y la gestión ambiental. Aportan elementos para la planificación del uso del suelo, en aspectos relacionados con la provisión de alimentos, energía, agua, disposición de residuos de una ciudad determinada, como es el caso de Manizales. En este texto, se presenta, en la primera parte, el cálculo de la Huella Ecológica de la ciudad de Manizales y la Biocapacidad BC del departamento de Caldas. En segundo lugar, se estima el déficit o superávit ambiental. Y por último, a manera de conclusiones, se proponen lineamientos de política para mejorar la gestión del territorio del departamento de Caldas y la calidad de vida de la población de Manizales para garantizar la sustentabilidad ambiental en la región.

\section{PALABRAS CLAVES}

Gestión ambiental, déficit ambiental, Huella Ecológica, Biocapacidad, Manizales.

\section{ECOLOGICAL FOOTPRINTS AND BIOCAPACITY: BIOPHYSICAL INDICATORS FOR ENVIRONMENTAL MANAGEMENT. MANIZALES'S CASE, COLOMBIA}

\begin{abstract}
The well-being of people located in a territory (a country, a city, a town) is intimately bound to the capacity of goods and environmental services available. Knowing the population's requirements and the capacity of the available space for the satisfaction of their necessities is fundamental to guarantee environmental sustainability. Ecological Economy allows us, by calculating the Ecological Footprint EF and Biocapacity BC, to
\end{abstract}


estimate the environmental deficit of the territory. Although at a global level the practical utility of these indicators for decision-making is limited, in the national, regional or local environments, they are useful instruments for decision-making in environmental planning and management; since they offer elements for the planning of land use, in aspects related to the provision of food, energy, water, housing and disposition of wastes of a city, like Manizales. This text presents in the first part, the calculation of Manizales' Ecological Footprint and Caldas' Biocapacity BC. In second place, the city's environmental deficit is considered. And finally, policies to improve the territorial management of Caldas and the well-being of Manizales' population are proposed in order to guarantee the environmental sustainability in the region.

\section{KEY WORDS}

Environmental management, environmental deficit, Ecological Footprint, Biocapacity, Manizales.

\section{INTRODUCCIÓN}

Desde cuando se dieron las primeras alarmas acerca de la insostenibilidad a que se exponía el planeta si se continuaba con el estilo de vida y el ritmo de consumo, los académicos y los políticos se han dado a la tarea de encontrar indicadores que permitan estimar la sostenibilidad de las economías. Así, desde la Economía Ambiental se han propuesto la contabilidad verde, el Producto Nacional Verde, el Ahorro genuino y el Índice de Desarrollo Económico Sustentable, como indicadores de "sostenibilidad". No obstante, dado que la Economía Ambiental parte del supuesto de que "el capital natural" puede ser sustituido por el "capital construido por los seres humanos", su alcance para dar cuenta de la sostenibilidad es limitado, y por ello se han considerado como indicadores de "sustentabilidad débill". En consecuencia, y desde la óptica de la Economía Ecológica se aportan indicadores alternativos como la Huella Ecológica, la Biocapacidad o el Espacio Ambiental, la Apropiación Humana de la Producción Primaria Neta (HANNP), Energy Return on Investment EROI, El MIPS o Mochila Ecológica, basados en flujos y consumos de energía y materiales que, a juicio de sus proponentes, se ubican en una concepción de "sustentabilidad fuerte", más acorde con la realidad biofísica del planeta.

No hay duda de que se ha avanzado en la construcción indicadores de "sustentabilidad fuerte (2) " como la Huella Ecológica o la Biocapacidad, pero aún estamos lejos de tener un indicador global que pueda dar cuenta de la (in)sostenibilidad del planeta. La HE, no obstante la dificultad que existe para su estimación (como ha sido señalado por los especialistas), es un indicador que a nivel global nos da una idea del "déficit ecológico" o el "superávit ambiental", partiendo del supuesto que los consumos son homogéneos, lo cual no es cierto, al menos, si consideramos y contrastamos los consumos promedio de los habitantes de los países desarrollados con los consumos per cápita de los países del Tercer Mundo. En este caso su utilidad práctica para la toma de decisiones es reducida o, incluso, puede asumirse solo como un ejercicio académico nada más. 
Quizá pueda ser un indicador útil para comparar "Huellas" entre países y, demandar a países con "Huellas" muy altas, la "Deuda Ecológica" que éstos tienen con países exportadores netos de materias primas, como es el caso de los países de nuestra región. También en los ámbitos local, regional o nacional puede ser un instrumento útil para la toma de decisiones de gestión ambiental en dichos niveles. Por ejemplo, sería una herramienta útil para tomar decisiones relacionadas con la planificación del uso del suelo o el espacio ambiental para proveer los alimentos, la energía, el agua, el espacio público y los espacios para la disposición de residuos de una ciudad o de una población determinada.

En esta perspectiva, en un ejercicio de investigación realizado con estudiantes de la Maestría en Medio Ambiente (3) , se ha calculado la Huella Ecológica de la ciudad Manizales y la Bicapacidad del Departamento de Caldas. El texto en su primera parte, discute las ventajas o limitaciones de estos indicadores como instrumentos para dar cuenta de la (in)sostenibilidad de una sociedad o un territorio, en segundo lugar presenta las estimaciones de la Huella Ecológica de la ciudad de Manizales y la Biocapacidad BC del departamento de Caldas. En tercer lugar, y con base en los resultados obtenidos, se estimó el déficit o superávit ambiental. Y, a manera de conclusiones y con base en los resultados anteriores, se sugieren lineamientos de política para mejorar la gestión del territorio y lograr la sustentabilidad de la región.

\section{La HE y la BC como indicadores de (in)sostenibilidad}

La $\mathrm{HE}$ es un indicador biofísico que nos permite estimar los requerimientos en términos de consumo de recursos y asimilación de desechos de una determinada población y economía, expresados en áreas de suelo productivo. Nos permite determinar la dependencia que tiene la población de una ciudad, región o país, de las importaciones de recursos y su capacidad de asimilación de desechos disponibles.

Según W. Rees y M. Wackernagel (1995), la Huella Ecológica HE, es definida como "el área o territorio ecológicamente productivo (cultivos, pastos, bosques o ecosistema acuático) necesaria para producir los recursos utilizados y para asimilar los residuos producidos por una población definida con un nivel de vida específico indefinidamente, donde sea que se encuentre esta área". En otras palabras, es un indicador biofísico del impacto de una población determinada, de acuerdo con su estilo de vida y la productividad de su espacio físico, dados por los consumos y la tecnología utilizada.

En principio, la HE parece un buen indicador de (in)sostenibilidad del planeta, una ciudad, una región o un territorio. De acuerdo al estilo de vida y de consumo de una sociedad, muestra el espacio en ha/per cápita requerida para satisfacer las necesidades de alimentación, oxígeno, energía, vivienda, agua, espacios para vertido, etc. La biocapacidad, por su parte, es la superficie de tierra disponible para un determinado nivel de producción y también se expresa en unidades de hectáreas globales. La diferencia entre biocapacidad y huella ecológica puede dar un excedente de recursos $(\mathrm{BC}-\mathrm{HE}>0)$ o un déficit ambiental $(\mathrm{BC}-\mathrm{HE}<0)$. Para el cálculo de la biocapacidad se requiere conocer la extensión de las tierras en producción y las que aún están desocupadas, con su rendimiento por unidad de área.

En síntesis, la HE contribuye a dar cuenta de la (in)sustentabilidad en la medida en que (4) : 
Agrupa en un solo dato el impacto que una determinada comunidad humana ejerce sobre los ecosistemas, tanto por el consumo de recursos como por la generación de residuos; permite definir y visualizar la dependencia de las sociedades humanas respecto al funcionamiento de los ecosistemas del planeta a partir de superficies apropiadas para satisfacer un determinado nivel de consumo; permite determinar el área productiva que una comunidad humana requiere, independientemente de que ésta se encuentre fuera de sus dominios; da la posibilidad de visualizar la inequidad social en la apropiación de los ecosistemas del planeta al realizar el cálculo para comunidades humanas o grupos sociales con consumos diferentes; y posibilita hacer el seguimiento del impacto de una comunidad humana sobre los ecosistemas, mediante la actualización del indicador a través del tiempo. Y en vista de la concentración de la población en las grandes ciudades, es posible hacer notar la dependencia que éstas tienen de los ecosistemas y del entorno.

Su cálculo parece elemental. No obstante, su estimación debe sortear varios escollos. El más importante es, en unos casos, la dispersión de datos y la falta de información confiable a nivel local, pues de muy poco sirve asumir consumos y producciones promedio tomados de otros contextos, para las estimaciones de los indicadores. En otros casos, su dificultad radica en la homogeneización de consumos entre estratos socioeconómicos con estilos de vida diferentes, caso que también ocurre para estimar la oferta disponible, debido a la diversidad de tecnologías aplicadas a los diferentes sistemas de producción.

La metodología de cálculo de la huella ecológica se basa en la estimación de la superficie necesaria para satisfacer los consumos asociados con la alimentación, los productos forestales, gasto energético y ocupación directa del territorio. La Huella Ecológica está compuesta de seis usos exclusivos de la superficie bioproductiva del planeta que compiten mutuamente por el espacio biológicamente productivo disponible. Estas categorías se expresan en hectáreas globales estandarizadas y se suman para determinar la HE total de una población Así, los terrenos productivos que se consideran para el cálculo son: cultivos, pastos, bosques, mar productivo, terreno construido y espacio público y área de absorción de CO2. La cantidad teórica de hectáreas necesarias para mantener en funcionamiento un sistema se calcula a partir de una matriz que relaciona diversos tipos de consumo con el territorio ocupado como superficie productiva.

Para calcular estas superficies, se siguen dos pasos básicos: uno, estimar en unidades físicas el consumo de las diferentes categorías para la población estudiada, y dos, con base en los requerimientos de cada categoría, calcular la superficie biológica productiva apropiada de acuerdo a los índices de productividad. Una vez calculados los consumos medios por habitante de cada producto, se transforman en área apropiada o huella ecológica para cada producto. Ello equivale a calcular la superficie necesaria para satisfacer el consumo medio por habitante de un determinado producto. La HE total por habitante, expresada en hectáreas por habitante, es el resultado de la matriz en superficie productiva, dividida por la población considerada $\underline{(5)}$.

Esta situación de demanda de recursos, puede ser "leída" también como los espacios necesarios para la generación de determinados productos, espacios necesarios básicamente para la utilización o demanda de ciertos recursos. Es dable inferir, que en este sentido, muchas regiones no son "autosuficientes" y demandan recursos de su 
entorno. Esto es claro en la comprensión del funcionamiento de las ciudades. Los límites urbanos de ellas, circunvalan un sistema interno que se haría insostenible, si de manera recurrente no entrasen y saliesen, materiales y energía, en distintos niveles de transformación que aseguran su metabolismo. La ciudad no es sostenible, sino fuese por su entorno. Pero por otro lado, la ciudad "impacta" sobre el entorno, de forma más o menos intensa. Y esta intensidad, está dada, en buena medida, por las formas de consumo de sus habitantes. En definitiva por el estilo de vida y los modelos de desarrollo imperantes.

\section{La huella ecológica de la ciudad de Manizales}

A excepción de Barcelona (6) y el Valle de Aburrá (7) , la HE ha sido poco calculada para ciudades. En este trabajo se calcula la Huella de Manizales (Caldas), una ciudad que concentra cerca del $40 \%$ de la población de departamento de Caldas. 382.182 personas en un área de 439,36 Km2, o 43.936 hectáreas. Se justifica este ejercicio, en el hecho de que el desarrollo de las actividades citadinas, demanda el aprovisionamiento de recursos que se producen en el entorno rural, tales como los alimentos, el agua, la energía, las materias primas para la construcción, el vestido, la producción de oxígeno, los espacios para la disposición de residuos sólidos, etc. Lo anterior ratifica la dependencia de la especie humana respecto de la naturaleza, de la cual los seres humanos no pueden prescindir. Ese espacio físico productivo requerido para satisfacer las demandas de la población de la ciudad es la "Huella Ecológica" de la ciudad, que presentamos a continuación.

Como ya se ha dicho, no existe toda la información requerida para estimar el indicador o la que se encuentra está dispersa y fragmentada en muchas instituciones. En consecuencia, este indicador se calcula tomando como referente la información disponible localmente e información nacional cuando fue necesario, lo que de alguna manera afecta la calidad del indicador y su utilidad con fines de diseño de políticas de gestión ambiental territorial. Pese a todo, es una buena aproximación que debe ser considerada por los tomadores de decisión y los gobiernos local y departamental.

En cuanto a las fuentes de información utilizadas, se puede mencionar el informe sobre la región Centro Sur, de la Secretaría de Agricultura del Departamento de Caldas. En éste documento se relaciona un inventario de oferta de bienestar (Alimentos, servicios, vías y espacios entre otros), para los municipios de Neira, Villamaría, Manizales, Palestina, Chinchiná. Además, la Secretaría de Planeación Municipal, el Ministerio de Agricultura y Desarrollo Rural, Fendipetróleos, Central Hidroeléctrica de Caldas, Aguas de Manizales, Corporación Colombia Internacional, Colanta, FAO Colombia, DANE, Unidad Municipal de Asistencia Técnica UMATA. Secretaria de Desarrollo Comunitario Municipio de Manizales, Secretaria de Agricultura de Caldas y Federación Nacional de Avicultores FENAVI. Los datos no disponibles, se complementaron en las matrices con los disponibles en las bases de datos de organizaciones públicas y privadas del orden nacional. Además de la información disponible en las instituciones, se recopiló información de primera mano para llenar los vacíos existentes, siguiendo los criterios metodológicos propuestos para otras ciudades por W. Rees y M. Wackernagel $\underline{(8)}$. 


\subsection{Cálculo de la Huella Ecológica}

Inicialmente, se definió una canasta de consumo de la población de Manizales. A partir de allí, se elaboraron las matrices de productos requeridos y de espacio físico necesario para garantizar dichos consumos per cápita. Se elaboraron tres matrices: La primera permite estimar el consumo energético, espacio público, vivienda y servicios públicos. La segunda se refiere al consumo de proteínas (carnes y huevo). La tercera resume los consumos de otros productos alimenticios (hortalizas, frutas, cereales y tubérculos). El consumo total (C) resulta de sumar la producción del departamento más las importaciones menos las importaciones (1)

Consumo ( C ) = Producción ( P ) + Importación ( I ) - Exportación ( E ) (1)

Tabla 1. Matriz síntesis: energía, vivienda y servicios. Año 2004

\begin{tabular}{|c|c|c|}
\hline $\begin{array}{l}\text { FUENTE } \\
\text { ENERGETICA }\end{array}$ & $\begin{array}{c}\text { FACTORES DE } \\
\text { EMISIÓN } \\
\text { (Kg CO2/Gj - } \\
\text { CONSUMOS P/C AÑO }\end{array}$ & $\begin{array}{c}\text { AREA DE ABSORCION DE } \\
\text { CO2 - AREA } \\
\text { REQUERIDA/hbt }\end{array}$ \\
\hline \begin{tabular}{|l} 
Combustibles \\
1. Fósil sólido \\
(Carbón) \\
2. Fósil líquido \\
(Gasolina, \\
Gasoil) \\
3. Gas
\end{tabular} & $\begin{array}{l}\text { (N. C.) }(9) \\
56.92 \\
146 \mathrm{M} 3\end{array}$ & $\begin{array}{l}\text { Hectárea por cada } 350.000 \text { hab. } \\
\qquad(0.03 \mathrm{Ha} / \mathrm{hab})\end{array}$ \\
\hline $\begin{array}{l}\text { Electricidad }(10) \\
\text { Biocombustibles } \\
(\mathrm{COOH}) \\
\text { Espacio Público }\end{array}$ & $\begin{array}{c}45 \\
2 \\
6 \mathrm{~m} 2 / \mathrm{hab}\end{array}$ & 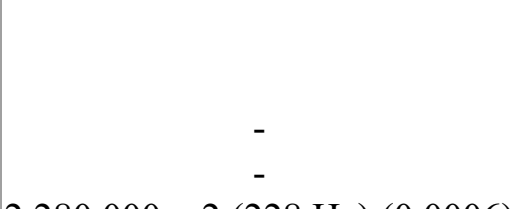 \\
\hline Vivienda & $7 \mathrm{~m} 3 / \mathrm{hab}$ & $\begin{array}{c}5.251 .331 .82 \mathrm{~m} 2(525.13 \mathrm{Ha}) \\
(0.027)\end{array}$ \\
\hline $\begin{array}{l}\text { Servicio público } \\
\text { Agua } \\
\text { Bosques } \\
\text { Pastos } \\
\text { Mar }\end{array}$ & $\begin{array}{l}\text { (N. C) } \\
\text { (N. C) } \\
\text { (N. C) }\end{array}$ & $\begin{array}{c}2.675 .351 \mathrm{~m} 3 \quad(267.5 \mathrm{Ha}) \\
(0.000704)\end{array}$ \\
\hline
\end{tabular}

Matriz 1:

Subtotal 1: $0.0583 \mathrm{Ha} \mathrm{p/c}$ anual 
Tabla 2. Consumo proteico: carnes, huevos, aceites y grasas. 2004

\begin{tabular}{|l|c|c|}
\hline \multicolumn{1}{|c|}{} & DEMANDA & AREA P/C REQUERIDA \\
\hline PRODUCTO & ANUAL P /C & EN HA. \\
\hline Carne Bobina & $19.8 \mathrm{Kg} \underline{(11)}$ & 0.264 \\
Carne Cerdo & $7.6 \mathrm{Kg} \underline{(12)}$ & 0.001085714 \\
Carne pollo & $17.73 \mathrm{Kg} \underline{(13)}$ & 0.0017729 \\
Pescado & $5.3 \mathrm{Kg} \underline{(14)}$ & 0.027 \\
Huevos & 384 unidades $\underline{(15)}$ & 0.0384 \\
Aceite (Soja, girasol, oliva, & & \\
otras semillas) & $33 \mathrm{Kg} \underline{(16)}$ & 0.004259 \\
\hline
\end{tabular}

Matriz 2

Subtotal 2: $0.336517 \mathrm{Ha} \mathrm{p/c}$

Tabla 3. Consumo de lácteos, cereales y hortalizas. 2004

\begin{tabular}{|l|c|c|}
\hline \multicolumn{1}{|c|}{} & DEMANDA ANUAL P & $\begin{array}{c}\text { AREA P/C REQUERIDA EN } \\
\text { PRODUCTO }\end{array}$ \\
\hline Arroz & HA. \\
\hline Papa & $45 \mathrm{Kg} \underline{(17)}$ & 0.009 \\
Plátano & $36 \mathrm{Kg}(18)$ & 0.00514 \\
Cebolla & $86.39 \mathrm{Kg}(19)$ & 0.0235 \\
Zanahoria & $0.20034 \mathrm{Kg}$ & 0.000265 \\
Tomate & $0.3075 \mathrm{Kg}$ & 0.0047 \\
Repollo & $0.7845 \mathrm{Kg}$ & 0.00384 \\
Banano & $0.2511 \mathrm{Kg}$ & 0.0031 \\
Piña & $0.1 \mathrm{Kg}(20)$ & 0.000030 \\
Papaya & $6.1 \mathrm{Kg}$ & 0.00014 \\
Naranja & $6.1 \mathrm{Kg}$ & 0.00024 \\
Cacao & $6.1 \mathrm{Kg}$ & 0.00056 \\
Lenteja & $1.2 \mathrm{Kg}$ & 0.00242 \\
Maíz & $3.5 \mathrm{Kg}$ & 0.01220 \\
Frijol & $33.1 \mathrm{Kg} \mathrm{(21)}$ & 0.03229 \\
Leche & $5.26 \mathrm{Kg}$ & 0.02221 \\
Queso & $65 \mathrm{Litros} \mathrm{(22)}$ & 1.6250 \\
Yogur & $3 \mathrm{Kg}$ & 0.3 \\
\hline
\end{tabular}

Matriz 3

Subtotal 3: $2.446 \mathrm{Ha} \mathrm{P/C}$

HE $=M 1+M 2+M 3=2.899$ hectáreas/per cápita/ año

\section{3 Cálculo de la Biocapacidad del departamento de Caldas}


El departamento de Caldas cuenta con un área de $7.507 \mathrm{Km} 2$, es decir 750.700 hectáreas. Según los estudios del Instituto Geográfico Agustín Codazzi IGAC (23) , el 14.1\% (110.321 hectáreas) del área son tierras aptas para el uso agrícola; $16.3 \%$ (122.609 has) son aptas para la ganadería; $65.4 \%$ (490.958 has) son tierras de vocación forestal; $2.9 \%$ (22.204 has) son suelos dedicados a áreas urbanas y vivienda rural; y el restante $2.3 \%$ (17.266 has) son áreas rocosas, eriales y nieves perpetuas, etc.

Como se puede apreciar, el espacio ambiental o la biocapcidad del departamento de Caldas, en su mayor parte está dedicado a la oferta de servicios ambientales (agua, captura de $\mathrm{CO} 2$ y productos del bosque) y producción de energía hidroeléctrica. El espacio disponible para la producción de alimentos tanto de origen vegetal como animal es mínimo. En consecuencia, la provisión de alimentos y servicios ambientales para su desarrollo es un asunto que amerita atención especial, ya que el bienestar de una población cualquiera está íntimamente ligado a la capacidad de su espacio ambiental para la provisión de dichos servicios.

En esta parte del trabajo se calcula la biocapacidad del departamento de Caldas, a partir de la información disponible en las instituciones que proveen los diferentes servicios en el departamento y en Manizales.

Tabla 4. Uso potencial del suelo en el Departamento de Caldas (Has.)

\begin{tabular}{|c|c|c|c|c|c|}
\hline Agrícola & Pecuario & Bosques & Vivienda & Otros & Total \\
\hline 110.321 & 122.609 & 478.300 & 22.204 & 17.266 & 750.700 \\
\hline
\end{tabular}

Fuente: elaborado con base en información del Atlas de Caldas (1987)

De acuerdo con estos datos, el suelo disponible o biocapacidad para satisfacer los requerimientos de la población del departamento de Caldas es de 733.434 hectáreas, que equivale a $1.93 \mathrm{ha} /$ persona/año. Si tenemos en cuenta que la Huella Ecológica, calculada con base en la canasta de consumo estimada, es de 2.899 has/persona/año, entonces la ciudad de Manizales tiene un déficit ambiental de: 0.969 has/persona/año

\section{BC-HE $<0$ déficit}

\section{Déficit Ambiental: 1.93- 2.899 = $\mathbf{- 0 . 9 6 9}$ has/persona/año}

Si bien la situación es preocupante debido al déficit resultante para satisfacer las necesidades de la población de la ciudad de Manizales que alberga solo el $40 \%$ de la población del departamento, lo que debe llamar la atención es la escasa disponibilidad de suelo para la producción de alimentos tanto de origen vegetal como animal. Para el primer caso, solo 110.000 hectáreas y para el segundo $122.000(24)$. Por supuesto, estos datos deben tomarse con beneficio de inventario dado que el uso real de los suelos no corresponde necesariamente con los estudios de uso potencial. De hecho, la producción de café en el departamento (95.000 has) se hace en suelos aptos para forestales. Igualmente, hay que considerar que buena parte de los suelos aptos para la producción agrícola como los de los Valles del Magdalena y el río Cauca están dedicados a la ganadería. 
Según datos de la Gobernación de Caldas, en el año 1996, 64.900 has estaban dedicadas a los cultivos de caña panelera, plátano, frutales, cacao, papa, yuca y maíz y fríjol; pasto natural 240.000, pasto artificial 160.000 y a bosques 127.000 has. Dichas cifras nos indican que hay una utilización inadecuada de los suelos. Muchos de los que deberían estar dedicados a los bosques y plantaciones forestales están dedicados a la ganadería extensiva, y algunos suelos agrícolas se dedican al rastrojo o a la ganadería.

Las anteriores estimaciones si bien nos muestran un panorama general de la utilización del espacio ambiental del departamento de Caldas, no son suficientes para sacar conclusiones definitivas en cuanto al déficit o superávit ambiental en el departamento de Caldas. Para tener una estimación más ajustada a la realidad es necesario calcular los déficit o superávit por categorías, y al interior de éstas por productos y servicios, de manera que se puedan plantear políticas específicas para cada caso. A continuación se presentan algunas estimaciones específicas:

\subsection{Cálculos para los principales servicios socioambientales}

\section{Servicio de Energía Eléctrica (BE)}

El consumo de energía eléctrica promedio para Manizales en el mes de enero de 2007, según la Superintendencia de Servicios Públicos Domiciliarios fue de 27.758,57 Mw. /hora (CE). La capacidad de distribución de energía eléctrica para el Departamento de Caldas es de $1.162 \mathrm{Mw}$. /hora (CDE) (25) ). La biocapacidad del departamento para el servicio energía eléctrica para el mes de enero de 2004 fue:

\section{Biocapacidad servicio energía eléctrica $(\mathrm{BE})=\mathrm{CDE}-\mathrm{CE}=1.162-27.758,57=$ - 26.596,57 Mw./hora}

Tabla 5. Consumo, capacidad y déficit energético de Manizales. 2007

\begin{tabular}{|c|c|c|c|c|c|c|}
\hline \multirow{2}{*}{ SERVICIO } & \multicolumn{2}{|c|}{ CONSUMO } & \multicolumn{2}{c|}{ CAPACIDAD } & \multicolumn{2}{c|}{ DÉFICIT } \\
\hline \begin{tabular}{c|c|c|c|c|c|} 
VALOR \\
(CE)
\end{tabular} & UNIDAD & $\begin{array}{c}\text { VALOR } \\
\text { (CDE) }\end{array}$ & UNIDAD & $\begin{array}{c}\text { VALOR } \\
\text { (BE) }\end{array}$ & UNIDAD \\
\hline $\begin{array}{c}\text { Energía } \\
\text { Enero 2007 }\end{array}$ & $27.758,57$ & Mw./hora & 1.162 & Mw./hora & $-26.596,57$ & Mw./hora \\
\hline
\end{tabular}

Fuente: Superintendencia de Servicios Públicos.

\section{Agua Potable (BAP)}

El consumo de agua potable promedio en Manizales para el año 2004 fue de 0.504 $\mathrm{m} 3 / \mathrm{seg}$. (CAP). El caudal promedio de las cuencas abastecedoras de agua del departamento para el mismo año, fue de $3.89 \mathrm{~m} 3 / \mathrm{seg}$. (QA). Con base en esta información, se estima el superávit para el año 2004:

$$
\begin{gathered}
\text { Biocapacidad servicio agua potable }(\text { BAP })=\mathrm{CA}-\mathrm{CAP}=3.89 \mathrm{~m} 3 / \mathrm{seg}-0.504 \\
\mathrm{~m} 3 / \mathrm{seg}=3.386 \mathrm{~m} 3 / \mathrm{seg}
\end{gathered}
$$


Tabla 6. Consumo y oferta hídrica en la ciudad de Manizales. 2004

\begin{tabular}{|c|c|c|c|c|c|c|}
\hline \multirow{2}{*}{ SERVICIO } & \multicolumn{2}{|c|}{ CONSUMO } & \multicolumn{2}{c|}{ CAPACIDAD } & \multicolumn{2}{c|}{ SUPERÁVIT } \\
\cline { 2 - 5 } & $\begin{array}{c}\text { VALOR } \\
\text { (CAP) }\end{array}$ & UNIDAD & $\begin{array}{c}\text { VALOR } \\
\text { (OA) }\end{array}$ & UNIDAD & $\begin{array}{c}\text { VALOR } \\
\text { (BAP) }\end{array}$ & UNIDAD \\
\hline $\begin{array}{c}\text { Agua } \\
\text { potable }\end{array}$ & 0.504 & $\mathrm{~m} 3 / \mathrm{seg}$ & 3.89 & $\mathrm{~m} 3 / \mathrm{seg}$ & 3.386 & $\mathrm{M} 3 / \mathrm{seg}$ \\
\hline
\end{tabular}

Fuente: Biomanizales. Observatorio para el Desarrollo Sostenible Manizales, Universidad Nacional de Colombia.

El cálculo anterior ratifica la oferta hídrica del departamento, si se tiene en cuenta que en el año 2004 solo se consumía el $12.96 \%$ de la oferta de este recurso. Sin embargo, es necesario actualizar la información con el fin de determinar posibles cambios en los caudales de reserva de las cuencas abastecedoras de agua y estimar los consumos por otro tipo de usos (industriales, agrícolas, etc.) no contemplados en el consumo doméstico, de tal manera que se tenga un dato de consumo más acorde con la realidad.

\section{Disposición Final de Residuos Sólidos Domésticos}

La producción de residuos sólidos en Manizales para el año 2004 fue en promedio de 274.92 Ton/día (26) . Si la densidad de los residuos sólidos es de 0.25 Ton $/ \mathrm{m} 3$, el volumen ocupado por los residuos sólidos de Manizales es de $1.099 .68 \mathrm{~m} 3$. De acuerdo con la fórmula Volumen $=$ Area*Altura y suponiendo una altura de montaña de residuos en el sitio de disposición final de $40 \mathrm{~m}$, se tiene que el área requerida para disponer los residuos sólidos de Manizales es de $27.49 \mathrm{~m} 2$ (ARS). Y dado que el relleno sanitario "La Esmeralda" cuenta con un área de $57 \mathrm{~m} 2$, se dispone de un espacio libre de cerca de $30 \mathrm{~m} 2$. En cuanto a la disposición de aguas residuales, para el año $2004 \underline{(27)}$ se estimó en $0.4032 \mathrm{~m} 3 / \mathrm{seg}$ (QAR). Dado que en la actualidad no se ejerce ningún tipo de tratamiento de los efluentes líquidos generados en la ciudad, debido a la inexistencia de Sistemas de Tratamiento de Aguas Residuales, no se calcula el espacio requerido para la disposición de éstas.

\section{Suelo urbano para la provisión de vivienda}

Según la Ley 388 de 1997, la Administración Municipal, a través de la formulación del Plan de Ordenamiento Territorial POT, debe generar políticas que definan la función del gobierno local y del sector privado en la provisión de alojamiento y servicios para la población que padece carencias habitacionales. La política de vivienda debe tener como objetivo mejorar las condiciones de vida de la población, a través del análisis de la problemática habitacional, del cálculo de las inversiones requeridas y asignación de responsabilidades institucionales.

Manizales, en la actualidad, mediante el acuerdo 508 de 2001, tiene disponibles 309 hectáreas como zonas aptas para el desarrollo (ZA) proyectadas al año 2008. No obstante, un estudio de la Secretaría de Planeación Municipal determinó que, solo el $62.4 \%$ de esa área (192.33) hectáreas, son realmente aptas para el desarrollo urbano. 
De otra parte, en el artículo $11^{\circ}$ del acuerdo 508 de 2001, se determinó que durante la vigencia del Plan de Ordenamiento Territorial, no se destina ninguna porción del territorio municipal como suelo de expansión urbana; y en su parágrafo $1^{\circ}$ expone que para definir la incorporación de áreas del suelo rural y/o del suelo suburbano como suelo de expansión urbano, deberá iniciarse un procedimiento de revisión del Plan de Ordenamiento Territorial. Igualmente se establece en el Decreto 879 de 1.998 en su Artículo 9, en su parágrafo $2^{\circ}$, que el cambio en la clasificación del Suelo Rural o Suelo Suburbano a Suelo de Expansión, deberá ser aprobado mediante acuerdo del Concejo Municipal. En el siguiente cuadro se presentan las necesidades de vivienda urbana en Manizales, así:

Tabla 7. Requerimientos de vivienda urbana de Manizales

\begin{tabular}{|c|c|c|c|c|}
\hline Suelo & $\begin{array}{c}\text { población } \\
\text { involucrada }\end{array}$ & $\begin{array}{c}\text { No. de viviendas } \\
\text { para } \\
\text { rehabilitación }\end{array}$ & $\begin{array}{c}\text { No. de } \\
\text { viviendas para } \\
\text { reubicación }\end{array}$ & $\begin{array}{c}\text { Viviendas } \\
\text { nuevas }\end{array}$ \\
\hline Urbano & 27.407 & 5.034 & 2.243 & 1802 \\
\hline
\end{tabular}

Fuente: Secretaría de Planeación Municipal, Oficina para la Prevención y Atención de Desastres OMPAD y Caja de la Vivienda Popular de Manizales.

\section{Área rural}

En el siguiente cuadro se presentan las necesidades de vivienda en el área rural de Manizales, así:

Tabla 8. Necesidades de vivienda en la zona rural de Manizales

\begin{tabular}{|c|c|c|c|c|}
\hline Suelo & Rehabilitación & $\begin{array}{c}\text { Viviendas } \\
\text { Nuevas }\end{array}$ & Reubicación & Mejoramientos \\
\hline Rural & 105 & 202 & 40 & 1.670 \\
\hline
\end{tabular}

Fuente: La Secretaría de Planeación Municipal, La Oficina para la Prevención y Atención de Desastres (OMPAD) y La Caja de la Vivienda Popular de Manizales.

\section{Resumen del déficit de vivienda}

El déficit habitacional total en el municipio de Manizales se presenta en el siguiente cuadro

Tabla 9. Síntesis del déficit de vivienda de Manizales

\begin{tabular}{|c|c|c|c|c|}
\hline Área & $\begin{array}{c}\text { Rehabilitación } \\
(28)\end{array}$ & Reubicación (29) & Nuevas & Mejoramiento \\
\hline
\end{tabular}




\begin{tabular}{|c|c|c|c|c|}
\hline Urbana & 5.034 & 2.243 & 1.802 & 4.850 \\
\hline Rural & 105 & 40 & 202 & 1.670 \\
\hline Total & 5.139 & 2.283 & 2.004 & 6.520 \\
\hline
\end{tabular}

Fuente: Secretaría de Planeación Municipal, Oficina para la Prevención y Atención de Desastres (OMPAD) y Caja de la Vivienda Popular de Manizales.

De acuerdo con las proyecciones realizadas, se trazan las siguientes metas a cumplir en la vigencia del presente Plan de Ordenamiento, en las zonas urbana y rural del municipio, para cada uno de los períodos del plan (corto, mediano y largo).

Tabla 10. Plan de vivienda para Manizales

\begin{tabular}{|c|c|c|c|}
\hline & $\begin{array}{c}\text { CONSTRUCCIÓN } \\
\text { DE VIVIENDA } \\
\text { NUEVA (UN) }\end{array}$ & $\begin{array}{c}\text { MEJORAMIENTO } \\
\text { URBANO (UN) }\end{array}$ & $\begin{array}{c}\text { MEJORAMIENTO } \\
\text { RURAL (UN) }\end{array}$ \\
\hline $\begin{array}{c}\text { CORTO } \\
\text { PLAZO }\end{array}$ & 1.016 & 1.000 & 600 \\
\hline $\begin{array}{c}\text { MEDIANO } \\
\text { PLAZO }\end{array}$ & 1.500 & 2.500 & 300 \\
\hline $\begin{array}{c}\text { LARGO } \\
\text { PLAZO }\end{array}$ & 1.832 & 1.500 & 300 \\
\hline
\end{tabular}

Fuente: Plan de Ordenamiento territorial 2005.

\section{Requerimientos de vivienda urbana en el municipio de Manizales 2.000- 2.024 (veinticinco años)}

Considerando: Una tasa de crecimiento de la población del 1\%, 3.5 personas por vivienda, 52.7 viviendas por hectárea, se tendría una densidad de 184.45 hab/ha.

Tabla 11. Área requerida para la provisión de vivienda 1999-2024

\begin{tabular}{|c|c|c|c|}
\hline \multicolumn{1}{|c|}{ Periodo } & $\begin{array}{c}\text { Déficit } \\
\text { acumulado en } \\
\text { No. de viviendas }\end{array}$ & $\begin{array}{c}\text { Población } \\
\text { proyectada }\end{array}$ & $\begin{array}{c}\text { Área (Ha) } \\
\text { Neta } \\
\text { Requerida }\end{array}$ \\
\hline A diciembre de 1.999 & $4.045^{*}$ & 363.739 & 76.81 \\
\hline A diciembre de 2.002 (3 años) & 7.194 & 374.761 & 136.5 \\
\hline A diciembre de 2.008 (9 años) & 18.559 & 397.816 & 352.2 \\
\hline A diciembre de 2.014 (15 años) & 20.774 & 422.290 & 394.2 \\
\hline A diciembre de 2.024 (25 años) & 32.078 & 461.853 & 608.7 \\
\hline
\end{tabular}

Fuente: Estimaciones con base en la información Secretaría de Planeación Municipal, Oficina para la Prevención y Atención de Desastres (OMPAD) y Caja de la Vivienda 
Popular de Manizales

Gráfico 1. Área requerida para construcción de vivienda 1999-2024.

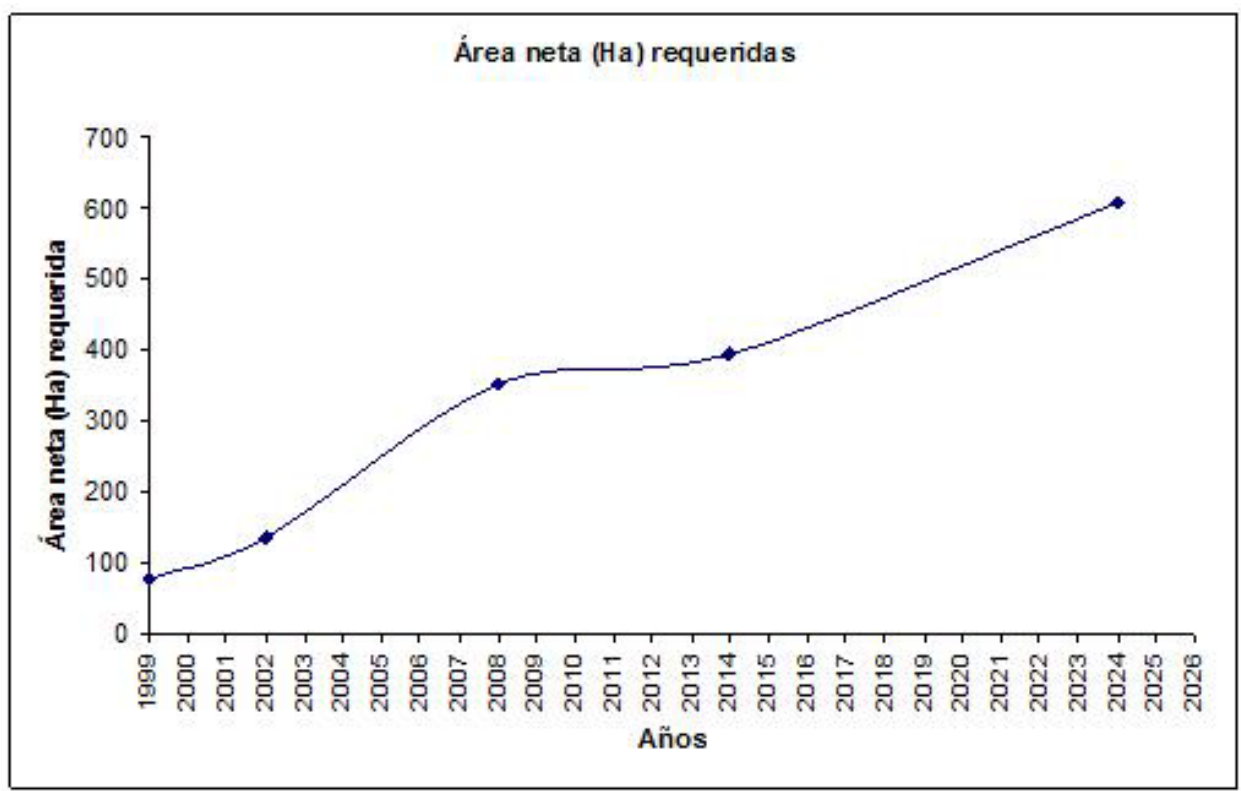

En el gráfico, se puede observar que entre 2003 y 2004 se comprometieron las 192.33 Ha, que el acuerdo 508/01 consideró aptas par el desarrollo. Por lo tanto el déficit para el 2007, en la ciudad de Manizales en cuanto a suelo urbano apto para el desarrollo es de aproximadamente 140 has.

Las posibles soluciones a esta problemática consisten en: i). La promoción y apoyo al desarrollo de programas de vivienda por medio de tratamientos de renovación urbana, redesarrollo y la densificación en sectores de localización estratégica o con buena dotación de infraestructura y transporte y ii). La incorporación de terrenos del suelo rural como suelo de expansión urbana, para generar proyectos urbanísticos por medio de Planes parciales.

\section{Conclusiones}

La Huella Ecológica para Manizales, de acuerdo a la canasta considerada, se estima 2.899 hectáreas por persona año. De acuerdo a los estudios realizados, es una Huella que está por encima de la capacidad del planeta ( 2.1 has/persona/año), aunque por debajo de HE para otras ciudades y países del mundo. Probablemente no es un indicador exacto, pero salvando las dificultades que se tienen para su cálculo, nos muestra una tendencia que puede ser considerada a la ahora de adoptar políticas para la gestión ambiental en la ciudad de Manizales y en el departamento de Caldas.

De acuerdo con los cálculos realizados, el componente que contribuye a incrementar el indicador es el consumo de alimentos básicos. Para la ciudad y el departamento este 
dato es muy importante, dado que el área agrícola del departamento, en su mayoría, está dedicada al cultivo del café que no es un producto fundamental de la dieta de los colombianos. Productos que son muy importantes en la dieta como el arroz, el trigo, la papa, el maíz, las hortalizas en buena parte se importan de otros departamentos. En el caso del arroz, el departamento no cuenta con suelos aptos para su cultivo, en tanto que el trigo es importado de otros países (Matriz 3).

Quizá donde Manizales y el departamento de Caldas cuentan con una mejor dotación es en la captura de $\mathrm{CO} 2$, provisión de agua y generación de energía hidroeléctrica. En estos rubros, la región puede ser exportadora neta. Incluso, podría ofrecer posibilidades de vender bosques como sumideros de CO2. De todas formas, aunque en este aspecto el indicador es excedentario, preocupa el hecho de que la ganadería esté ocupando cada vez más áreas forestales y de conservación. Las autoridades y los gobiernos municipal y departamental deben adoptar medidas orientadas a revertir esta tendencia so pena de tener problemas de abastecimiento en el futuro.

El área total de Manizales, es de 439,36 Km2, o 43.936 hectáreas. Su población estimada para 2004 es de 382.193 habitantes y su huella per cápita es de 2.869 Ha. De esto se deduce que el Espacio Ambiental requerido para satisfacer los consumos de la ciudad es de 1.107.418 has. Es decir, 25 veces el área disponible para la provisión de bienes y servicios ambientales. Incluso, Manizales no puede satisfacer sus requerimientos con el área ambiental disponible del departamento de Caldas, sobre todo en suministro de alimentos y espacio para la vivienda urbana.

En este aspecto la ciudad presenta serias dificultades por insuficiente espacio para la provisión de vivienda y espacio público. Manizales es una de las ciudades con el menor espacio físico por habitante en Colombia. Esta situación, en parte, se debe a que la topografía donde se encuentra la ciudad es demasiado abrupta, lo cual hace que el área para la construcción de vivienda y la oferta de espacio público sean muy escasas. Las posibilidades de expansión urbana son mínimas, situación que afecta la disponibilidad de espacio público y pone en dificultad a la ciudad para satisfacer una demanda habitacional creciente.

La biocapacidad en relación con el suelo urbano y su aptitud social fundamental de proveer área neta efectiva para la dotación de vivienda resulta crítica tanto para la ciudad como para el departamento, entendiendo además que en la ciudad se presenta la mayor demanda en virtud de su déficit cualitativo y cuantitativo creciente y su condición de ciudad universitaria que atrae población de otros municipios y otros departamentos. En virtud de ello, es fundamental definir en el POT el suelo de expansión urbana, que permita mediante la aplicación de Planes Parciales, presentar la oferta de áreas aptas para vivienda con calidad urbanística y ambiental, y costos razonables.

Si no se adoptan las políticas adecuadas a la expansión urbana y a la provisión de alimentos, la ciudad se tornará en el corto tiempo insostenible, en términos de un concepto de sustentabilidad fuerte. La cuestión es evidente en el caso de vías, parques y zonas aptas para la construcción. En cuanto a alimentos, la ciudad depende de la importación de la mayoría, los cuales provienen, en primer lugar, de los municipios con los que comparte el concepto de "Región" y, en segundo lugar, de otros departamentos 
y de otros países. En consecuencia, el futuro de la ciudad se hace inviable, su desarrollo limitado y el bienestar de la población se afecta.

En lo que respecta a oferta hídrica y energética, históricamente el departamento de Caldas ha sido reconocido por su potencial hidroeléctrico y sus buenas condiciones de brillo solar. De todas formas, surge la necesidad de recabar información que permita determinar la capacidad urbana departamental para proveer energía a través de los ductos y redes de distribución del gas domiciliario, en donde la ciudad es deficitaria.

La capacidad hídrica estimada en este ejercicio se reduce a la disponibilidad hídrica superficial, la cual presenta excedentes para el abastecimiento futuro de la ciudad y los municipios vecinos, partiendo del supuesto de que se cuente con los recursos y una política adecuada para el manejo forestal, hidráulico y sanitario que demanda el suministro sostenible de agua potable para la ciudad de Manizales y su entorno departamental. Con relación a otras fuentes hídricas como las subterráneas se carece de información que dé cuenta de caudales y factibilidad de uso.

El Municipio de Manizales como tal presenta una considerable biocapacidad en términos de la disposición controlada de residuos sólidos en el Relleno Sanitario "La Esmeralda", máxime si se asume la expansión de su vida útil hasta el año 2.025, siendo receptor incluso de los residuos generados en otras municipalidades del Departamento de Caldas. No obstante, debería analizarse las consecuencias de los lixiviados y olores dado que el relleno es a cielo abierto.

Al revisar la capacidad de saneamiento hídrico de la ciudad en cuanto a las aguas residuales domésticas deberá considerarse la aplicación de Modelos de Simulación Limnológica y Sanitaria de las fuentes hídricas receptoras, que determinen con mayor certeza la biocapacidad de este servicio ambiental, en función de la capacidad autodepuradora de las quebradas y ríos que sirven como colectores primarios de la carga contaminante combinada arrojada diariamente (SST + DBO5)

La Biocapacidad del suelo rural se puede hacer más eficiente mediante la planificación del uso acorde con la aptitud de la tierra o el uso potencial del suelo para actividades productivas de tipo agropecuario, forestal y minero. En este sentido, se requiere investigación para mejorar la productividad por unidad de área, de manera de hacer más eficiente el uso del recurso suelo. Igualmente, se requiere una política de aprovechamiento, restauración y conservación de las áreas con potencial forestal, que permita mantener y ampliar la oferta de servicios ambientales estratégicos para el globo, como la captura de $\mathrm{CO} 2$, la producción de agua y el mantenimiento de la biodiversidad, y otros servicios vitales para la matriz ciudad- departamento.

Por último, la $\mathrm{HE}$ y la $\mathrm{BC}$, como indicadores biofísicos de (in)sostenibilidad y autosuficiencia de una población y espacio determinados, contribuye a que los actores locales, los gobernantes, tomadores de decisiones, adopten comportamientos y diseñen políticas de consumo y uso del espacio ambiental, orientados a garantizar la sostenibilidad ambiental en el tiempo. En lo concerniente a la provisión de alimentos es posible plantear alternativas de producción que permitan orientar la gestión local hacia el logro sociedades sostenibles. Lo anterior daría la posibilidad de generar una política de seguridad alimentaria local no dependiente de ofertas externas, sujetas a las libres 
fuerzas del mercado, salvo para aquellos productos donde la oferta ambiental no esté disponible.

El ejercicio realizado es interesante pero tiene muchos escollos que son difíciles de sortear. Uno de ellos es la falta de información confiable a nivel local, pues de muy poco serviría asumir consumos y producciones promedio tomados de otros contextos, para el caso de la HE. En cuanto a la BC, ocurre algo similar. La información está dispersa y la que tienen las diferentes entidades muchas veces no es confiable. Esta apreciación no debe tomarse como factor negativo; por el contrario, debe servir de advertencia a la comunidad para que tome conciencia del estado de su ciudad. En síntesis, el cálculo de la Huella Ecológica de Manizales y la Biocapacidad del departamento de Caldas, es una tarea aún inacabada que, debido a que los consumos y el desarrollo tecnológico cambian con el tiempo, es necesario calcularla y actualizarla periódicamente.

\section{BIBLIOGRAFÍA}

- Agudelo Patiño, Luis Carlos. "Indicadores de sostenibilidad y ordenación del territorio. Huella Ecológica y ecosistemas estratégicos en Medellín, Colombia". www.docentes.unal.edu.co/.../docs/INDICADORES.

- Alcaldía de Manizales. (2005). Proyecto Plan de Desarrollo 2005 - 2007. ESAP Mesas de Trabajo.

- Alcaldía de Manizales. (2004). Planes de Desarrollo 1995 - 1997. 2000 - 2003 Plan de Inversión social Manizales 2004.

- FEDERACAFE. (2002). Gerencia Financiera y de Investigaciones Económicas. Censo Agrocafetero 2001.

- FENAVI. (2005). Informe anual producción exportación y consumo de pollo y huevos.

- Gachet Otañez, Iván Francisco. (2002). La huella ecológica: teoría y método y tres aplicaciones al análisis económico.

- Gobernación de Caldas. (1996). Carta Estadística. Imprenta Departamental. Manizales. Ediciones Abya-yala. Quito (Ecuador).

- Ministerio de Agricultura. Gobernación de Caldas. (1987). Atlas de Caldas. Imprenta Departamental. Manizales (Colombia).

- Rees, William E. (1996b). "Indicadores territoriales de sustentabilidad". En: Ecología Política. Núm. 12. Barcelona, pp. 27-41.

- Molina Poveda, Yolanda y Yepes Pérez, Fabio. (1999a). "La huella ecológica: indicador de sustentabilidad en la planificación ambiental de cuencas hidrográficas". En Naturaleza y Sociedad. Núm. 2. Ibagué (Colombia), pp. 4777.

- Relea Ginés, Ferrán. “Aproximación de la Huella Ecológica de Barcelona”. Resumen de los cálculos y reflexiones sobre losresultados. www.bcn.es/mediambient/cas/down/masu6 1.pdf.

- Wackernagel, Mathis. (1996b). "Ciudades sostenibles". En Ecología Política. Núm. 12. Barcelona, pp. 43-50.

- Wackernagel, Mathis and Rees, WilliamE. (1995). Our Ecological footprint: Reducing Human Impact on the earth. Gabriola Island, BC and Philadelphia, 

PA.
New
Society
Publishers.

www.cfnavarra.es/medioambiente/agenda/Huella/EcoSos.htm

\section{NOTAS}

1. Este texto es una reflexión, a partir de los cálculos de la Huella Ecológica HE y la Biocapacidad $\mathrm{BC}$, realizada con un grupo de estudiantes de la maestría en Medio Ambiente y Desarrollo de la Universidad Nacional de Colombia sede Manizales, durante los años 2006 y 2007.

2. La Sustentabilidad fuerte es un indicador en el cual los recursos naturales no pueden ser sustituidos por recursos producidos por los seres humanos. El proceso económico requiere un stock de "capital natural" sin el cual la economía no funcionaría.

3. Promociones 2005a y 2006 b de la Universidad Nacional de Colombia sede Manizales, Colombia.

4. http://www.cfnavarra.es/medioambiente/agenda/Huella/EcoSos.htm. Consultado en agosto de 2007.

5. http://www.cfnavarra.es/medioambiente/agenda/Huella/EcoSos.htm. Consultado agosto de 2007.

6. Relea Ginés, Ferrán. Aproximación de la Huella Ecológica de Barcelona. Resumen de los cálculos y reflexiones sobre los resultados. www.bcn.es/mediambient/cas/down/masu6_1.pdf. Consultado diciembre de 2007.

7. Agudelo Patiño, Luis Carlos. Indicadores de sostenibilidad y ordenación del territorio. Huella Ecológica y ecosistemas estratégicos en Medellín, Colombia. www.docentes.unal.edu.co/.../docs/INDICADORES. Consultado diciembre de 2007.

8. Generada por hidroeléctricas. Constituyen energía renovable.

9. No incluido en la canasta consultada. El consumo se ha reemplazado por gas natural domiciliario.

10. Central de Sacrificio de Manizales. Rendimiento promedio/ unidad sacrificada $150 \mathrm{Kg} / 3$ Unidades por Ha.

11. Central de Sacrificio de Manizales. Rendimiento promedio/ unidad sacrificada $70 \mathrm{Kg}$. /100 unidades por Ha.

12. FENAVI. Dado que no se encuentra el consumo para Manizales, se toma el promedio de demanda nacional. I

13. Umata. Secretaria de Desarrollo Comunitario Alcaldía de Manizales. Rendimiento estimado por Ha. $800 \mathrm{Kg}$.

14. FENAVI. Se asume el promedio de consumo nacional.

15. Rendimiento estimado 7.747 litros/ha/año.

16. FEDEARROZ. Consumo y producción promedio nacional.

17. FEDEPAPA. Consumo y producción promedio nacional.

18. Centro Comercial Galerías. Umata, Manizales. Consumo promedio para Manizales y producción promedio de Caldas, para Plátano, Cebolla, Zanahoria, Tomate y Repollo.

19. FAO Colombia, Ministerio deAgricultura, Federación Cacaoteros. Información nacional para banano, piña, papaya, naranja, cacao y lenteja 
20. Federación Nacional de cultivadores de Cereales FENALCE. Información para maíz y fríjol.

21. Cooperativa de Lecheros de Antioquia COLANTA. Información para leche, queso y yogur.

22. Ministerio de Agricultura. Gobernación de Caldas. Atlas del departamento de Caldas. Manizales. Imprenta departamental, 1987.

23. Ministerio de Agricultura-Gobernación de Caldas. Op.cit.

24. Página Web de la CHEC.

25. Dato tomado de la base de indicadores del Biomanizales. Observatorio para el Desarrollo Sostenible Manizales, Universidad Nacional de Colombia.

26. Suponiendo que el caudal de agua residual es el $80 \%$ del consumo de agua potable para el mismo periodo.

27. La rehabilitación consiste en el mejoramiento de la vivienda en el mismo sitio.

28. Implica construir nuevas viviendas en un lugar diferente a donde se encuentra la vivienda. Manizales debido a su topografía tiene muchas viviendas en zonas de alto riesgo y vulnerabilidad que requieren ser reubicadas. 